\title{
Solid plexiglass clips to induce reproducible renal hypertension in the rat
}

\author{
A.A. Nekooeian, T. Mashhoodi
}

Department of Pharmacology, Shiraz Medical School, Shiraz University of Medical Sciences, Shiraz, Iran

Received: 11.7 .2005

Revised: 29.9.2005

Accepted: 10.10 .2005

Correspondence to:

Ali Akbar Nekooeian E-mail: nekooeiana@sums.ac.ir

\section{Introduction}

The two-kidney, one-clip renovascular hypertension was first described by Goldblatt $e t{ }^{2}{ }^{[1]}$ who demonstrated that partial constriction of the renal artery with an adjustable silver clip in dogs led to persistent hypertension. Since then hypertension in rats has been achieved by clamping renal artery by adjustable silver clips. The adjustable silver clips suffer from certain disadvantages, like variations in silver flexibility leading to poor fabrication and less uniformity in adjustable clips compared to fixed-size silver clip ${ }^{[1]}$ and procuring difficulties from far away manufacturers. Therefore the present study was undertaken with the objective to induce renal hypertension by clips made from inexpensive and easyto-obtain material like polymethyl methacrylate (plexiglass).

Clips with an internal diameter of $0.20-0.21 \mathrm{~mm}$, was made from a piece of $2 \mathrm{~mm}$ thick plexiglass. Using a saw blade (0.21-0.22 mm thick), $3 \mathrm{~mm}$ deep slits were made. Clips were then cut and separated in rectangular pieces $([4 \times 2 \times 2 \mathrm{~mm}]$ Figure 1$)$. The internal diameter of silver clips (a courtesy of Dr. Mohammad Ali Sharifi, Iran University of
Medical Sciences, Tehran, Iran) was $0.2 \mathrm{~mm}$. Three groups ( $n=6$ each) of male Sprague-Dawley rats (200-250 g) consisting of a (1) sham, (2) a renal artery (left renal artery) clipped with plexiglass clips and (3) a renal artery clipped with silver clips were included for the study. Under xylosine $(8 \mathrm{mg} / \mathrm{kg})$ and ketamine $(60 \mathrm{mg} / \mathrm{kg})$ anesthesia, (i.p.) animals were subjected to sham surgery or placement of clips around left renal artery. Briefly a $2 \mathrm{~cm}$ incision was made in the left flank and left kidney was exposed. Then the left renal artery was separated from left renal vein and surrounding tissues. Afterwards, a clip was placed around renal artery as close as possible to the aorta. After recovery from anesthesia, each animal was housed in a single cage. Four weeks after the surgical procedure, (MBP) systolic and diastolic pressures and heart rate (HR) were measured under thiobutabarbital Inactin $(100 \mathrm{mg} / \mathrm{kg})$ anesthesia. Afterwards, they were sacrificed by a bolus of anesthetic thiobutabarbital and the weights of heart (HW), left kidney (LKW) and right kidney (RKW) were determined and were normalized to body weight. Mean blood pressure (MBP) was calculated as diastolic pressure 
Figure 1: A plexiglass clip with a magnification of $10 x$

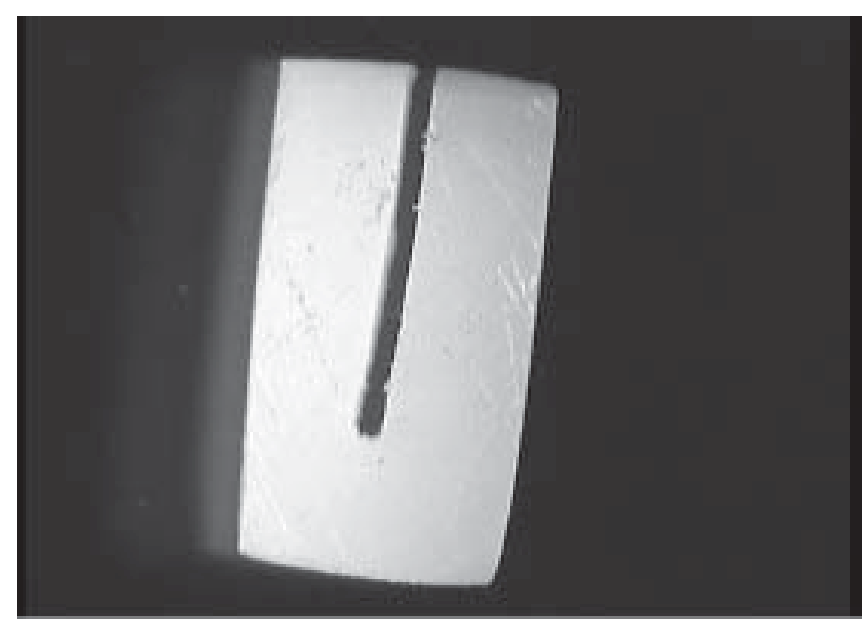

plus one third of pulse pressure. The data, presented as Mean \pm SD, were analyzed using one way analysis of variance followed by Tukey test. $P \leq 0.05$ was considered significant.

There was no mortality in any of the groups. Body weights on the day of operation (BWO) or on the day of experiment (BWE) of sham-operated rats (SOR), renal artery clamped rats with plexiglass clips (RACRPC) and renal artery clamped rats with silver clips (RACRSC) were not significantly different. Moreover, there was no significant difference in the HR among the groups. The RACRPC and RACRSC groups had significantly higher MBP, HW and RKW and a significantly lower LKW. There was no significant difference in the values of HR, MBP, HW, RKW, LKW, BWO or BWE between RACRPC and RACRSG groups [Table 1].

The cost and time to purchase the adjustable silver clips from abroad, along with the difficultly in reproducibility and consistency of hypertension induced by this type of clips ${ }^{[2,3]}$ prompted us to try to induce hypertension by easy-to-get means. We did try to make solid silver clips using a silver bar. However, the use of solid silver clips were with many limitations. Firstly, the hardness or softness of silver bars depended on the carat of silver in the alloy and ranged from very soft to very hard. Obtaining the most appropriate alloy with a favorable hardness required repeated visits to the silversmith, which did not seem justified in terms of the time spent. Secondly, we found sawing of the silver bar quite difficult, which was limted by the hardness of the silver bar. Thirdly, a solid silver clip was found to be much heavier than a solid plexiglass clip with an identical size. Consequently, we were not sure that such a heaviness would not lead to falling off of clips placed around renal artery or would not lead to failure. We therefore decided to make clips using something other than silver bar. We found that plexiglass clips with the above-

\section{Table 1}

Values of heart rate (HR, beat/min), mean arterial pressure (MAP, $\mathrm{mmHg}$ ), heart weight (\% body weight), left kidney weight (LKW, $\%$ body weight) right kidney weight (RKW, \% body weight), body weight on the day of operation (BWO, g) and body weight on the day of experiment day (BWE, g) of sham-operated and left renal artery clipped rats using plexiglass or sliver clips at 4 weeks after sham-operation or renal artery clipping

\begin{tabular}{lccc}
\hline & Sham & Plexiglass clip & Silver clip \\
\hline HR (beats/min) & $362 \pm 32$ & $412 \pm 23$ & $426 \pm 18$ \\
MAP (mmHg) & $103 \pm 7$ & $173 \pm 3^{*}$ & $172 \pm 7^{*}$ \\
HW (\% of body weight) & $0.28 \pm 0.01$ & $0.38 \pm 0.01^{*}$ & $0.39 \pm 0.04^{*}$ \\
LKW (\% of body weight) & $0.37 \pm 0.02$ & $0.27 \pm 0.02^{*}$ & $0.28 \pm 0.02^{*}$ \\
RKW (\% of body weight) & $0.37 \pm 0.01$ & $0.46 \pm 0.03^{*}$ & $0.49 \pm 0.02^{*}$ \\
BWO (g) & $233 \pm 9$ & $240 \pm 10$ & $229 \pm 12$ \\
BWE (g) & $289 \pm 20$ & $271 \pm 16$ & $272 \pm 11$ \\
\hline
\end{tabular}

Values $(n=6)$ are as Mean $\pm S D$, Denotes significantly different from shamoperated group

mentioned dimensions could induce hypertension in which values of MBP, HW, RKW and LKW were comparable to those in hypertension induced by silver clips. ${ }^{[4]}$ The fabrication of solid plexiglass elips is cheaper by cost and can be done in any pharmacology or physiology lab using simple and much less sophisticated equipments. Low cost and reproducibility of plexiglass clip-induced hypertension provide a favorable opportunity to induce experimental renal hypertension and for preclinical evaluation of new antihypertensives.

\section{Acknowledgements}

This work was supported by Vice Presidency in Research Affairs, Shiraz University of Medical Sciences.

\section{References}

1. Goldblatt H, Lynch J, Hanzal RF, Summerville WW. Studies on experimental hypertension, I. The production of persistent elevation of systolic blood pressure by means of renal ischemia. J Exp Med 1934;59:347-50.

2. Kivilghn SD, Zingaro GJ, Gabel RA, Broten TP, Siegl PK. Models of experimental hypertension. In: McNeill JH. Measurement of cardiovascular function. CRC Press: New York; 1997. p. 69-87.

3. Dussaule JC, Michel JB, Auzan C, Schwartz K, Corvol P, Menard J. Effects of antihypertensive treatment on the left ventricular isomyosin profile in one clip, two kidney hypertensive rats. J Pharmacol Exp Ther 1986;236:512-8.

4. Hocher B, George I, Diekmann F, Zart R, Rebstock J, Schwarz A, et al. ETA receptor blockade induces fibrosis of the clipped kideny in twokidney-one-clip renovascular hypertensive rats. J Hypertension 2000;18:1807-14

Source of Support: Vice Presidency in Research Affairs, Shiraz University of Medical Sciences. Conflict of Interest: Nil. 